\title{
Structural design of parallel manipulators with general constraint one
}

\author{
Rasim Alizade*, Ozgun Selvi, Erkin Gezgin \\ Mechanical Engineering Department, Izmir Institute of Technology, Urla 35437, Izmir, Turkey
}

\section{A R T I C L E I N F O}

\section{Article history:}

Received 4 October 2008

Received in revised form 2 June 2009

Accepted 19 June 2009

Available online 12 October 2009

\section{Keywords:}

Overconstrained parallel manipulators

Linear-angular conditions

Recurrent vector equations

\begin{abstract}
A B S T R A C T
In this paper structural design of parallel manipulators with general constraint one regarding angular and linear-angular conditions are considered. Four known overconstrained mechanisms with angular and two new designs with linear-angular conditions are presented. 14 structural groups and end effector chains in subspace $\lambda=5$ are examined. New formulations and definitions of constructing overconstrained manipulators are described. Using examined structural groups, all architectures of subspace $\lambda=5$ parallel manipulators with revolute joints and single-loop are introduced via structural bonding.
\end{abstract}

(c) 2009 Published by Elsevier Ltd.

\section{Introduction}

Structural synthesis of overconstrained manipulators can be categorized as an important step of the general structural design study of fundamental mechanism and machine science. Structural design of overconstrained multi-mobility manipulators is a geometrical methodology that is used to generate all related architectures of the dedicated area. In order to widen the applications of overconstrained manipulators in industry, manipulator motions in subspaces should clearly be investigated.

Throughout the literature, several 3D overconstrained mechanisms with angular conditions have been discovered. Sarrus [1] described a special case of planar-hybrid linkage, which has six axes intersecting by pairs of three at distinct points, and Bennett [2] introduced a spherical hybrid linkage as well as a plano-spherical hybrid linkage with the criteria of intersecting six axes by pairs at two different points.

Seven different types of mobile 6R linkages with linear-angular conditions were discovered by Bricard [3]. By combining three Bennett loops with linear and angular conditions, two 6R overconstrained linkages are constructed by Goldberg [4]. As an inverse of Bricard's orthogonal 6R linkage, the "wirbelkette" overconstrained mechanism with equal link lengths and zero joint offsets was introduced by Franke [5]. Altmann [6] presented a 6R linkage, which is a special case of the Bricard line symmetric linkage. The general model of the six link mechanism with six skew orthogonal axes and equal link and offset lengths is described by Harrisberger and Soni [7]. Waldron [8-10] proposed a family of overconstrained hybrid linkages, where some of them are created by combining Bennett overconstrained linkages. Wohlhart [11,12] combined double Goldberg linkages to construct an overconstrained hybrid Bennett-based 6R linkages. Mavroidis and Roth [13] developed a 6R overconstrained mechanism with two Bennett joints that have no common axis. Dietmeier [14] introduced a new family of overconstrained 6R linkages.

A special trihedral Bricard linkage was derived by Schatz [15] and new asymmetrical 6R linkage has been obtained with single degree of mobility. The new RRRS symmetrical overconstrained mechanism with linear and angular conditions, and

\footnotetext{
* Corresponding author. Tel.: +90 232750 6544; fax: +90 2327507890 .

E-mail addresses: rasimalizade@iyte.edu.tr, alizada_rasim@hotmail.com (R. Alizade).
} 
another new overconstrained mechanism with linear and angular conditions in subspace $\lambda=5$ are described by Alizade et al. [16]. Only a few researchers, Baker [17-22], Mavroidis et al. [13,23-26], Karger [27], Shih and Yan [28], Lerbet [29], Jin and Yang [30], deal with analytical methods. In the studies of Baker [17-23], both the analysis of the overconstrained Altmann's linkage by using geometric and algebraic way. Moreover, Dai et al. [31] propose a new approach to mobility analysis based on the motion decompositions and constraints in screw systems. The traditional mobility rules for a linkage is included and strengthened with a new equation by Guest and Fowler [32] and applied to the overconstrained mechanisms. Furthermore, Huang and Sun [33] investigated the finite displacements of all known Bennett-based 6R overconstrained linkages by the help of numerical simulations and found that every Bennett-based 6R linkage, except for the isomerization of Wohlhart's hybrid linkage, relates with the properties of the Bennett mechanism.

A parallel manipulator can be defined as a platform connected to the ground by at least two legs and motors that are distributed to these legs. One of the investigations about parallel manipulators with two legs is done by Li et al. [34]. The study is related to the 2 DoF parallel manipulator with spherical output and its work space analysis. The only investigation known to us about overconstrained parallel manipulators with two legs is done by Gogu [35]. An approach for structural synthesis of overconstrained parallel wrists with 2 DoF has been proposed in the study. The main property of the designed overconstrained mechanism is its spherical output with a singularity free and fully isotropic structure.

In this paper the new formulated recurrent unit vector equations are used for describing the orientation of links in mechanisms. The analytic approach helped us to create linear and angular conditions for two kinds of RRRS mechanisms with onegeneral constraint. Further the theory of structural groups with one-general constraint is introduced. Later the mobility of the end effector chains of the rigid body in subspace $\lambda=5$ is described. Finally the theory in the design of possible architectures of parallel manipulators with angular or linear-angular conditions are given. All this theory is presented by serial examples of new overconstrained parallel manipulators in subspace $\lambda=5$. These investigations permit to create parallel manipulators with general constraint one that are composed of single-loop and the link that describes the motion of the end effector in 3D.

\section{Recurrent unit vector equations}

Spatial serial or parallel manipulators consist of kinematic chains. In order to describe position and orientation of these links mathematically, different methods can be applied. The most commonly used orientation coordinates can be given such as Euler angles, Euler parameters, Rodriguez parameters and direction cosines. Being the part of these methods, recurrent unit vector equations are described by including link $\left(d_{j}, \theta_{j}\right)$ and kinematic pair $\left(a_{j}, \alpha_{j}\right)$ parameters by Denavit and Hartenberg [36]. Note that recurrent unit vectors are the unit vectors, where their directions are intersected by one another in series. The unit vector equations are derived to find the direction of the third vector with respect to any reference frame by giving the directions of two vectors in that reference frame.

As shown in Fig. 1 three independent unit vectors $\mathbf{e}_{i}, \mathbf{e}_{j}, \mathbf{e}_{k}$ describe the joint parameters as $d_{j}=a_{i k}$ and $\theta_{j}=\alpha_{i k}$ (Fig. 1a), and also the link parameters as $a_{j}=a_{i k}$, and $\alpha_{j}=\alpha_{i k}$ (Fig. 1b). In any case, two unit vectors $\mathbf{e}_{i}=\left[l_{i}, m_{i}, n_{i}\right]^{T}$ and $\mathbf{e}_{k}=\left[l_{k}, m_{k}, n_{k}\right]^{T}$ will describe the directions of links for joint parameters and the directions of joints for link parameters. The directions of joint and link are a unit vector, $\mathbf{e}_{j}=\left[l_{j}, m_{j}, n_{j}\right]^{T}$ as shown in Fig. $1 \mathrm{a}$ and $\mathrm{b}$ respectively. Note that, for revolute pair, parameters $\alpha_{i k}$ is variable while $a_{i k}$ is constant, but for prismatic pair, while parameters $\alpha_{i k}$ is constant $a_{i k}$ is variable (Fig. 1a).

Now let the directions of two unit vectors $\mathbf{e}_{i}$ and $\mathbf{e}_{j}$ are known. Knowing the two directions, our problem is to compute the direction of the third unit vector $\mathbf{e}_{k}$. Throughout the solution of this problem, first we will describe the vector equations of the three unit vectors as,

$$
\mathbf{e}_{i} \times \mathbf{e}_{k}=\mathbf{e}_{j} \operatorname{Sin} \alpha_{i k}, \quad \mathbf{e}_{i} \cdot \mathbf{e}_{k}=l_{i} l_{k}+m_{i} m_{k}+n_{i} n_{k}=\operatorname{Cos} \alpha_{i k}, \quad \mathbf{e}_{j} \cdot \mathbf{e}_{k}=l_{j} l_{k}+m_{j} m_{k}+n_{j} n_{k}=0
$$

If the first equation of Eq. (1) is wanted to be introduced in algebraic form, it can be represented as

$$
\left(l_{j} \mathbf{i}+m_{j} \mathbf{j}+n_{j} \mathbf{k}\right) \operatorname{Sin} \alpha_{i k}=\left(m_{i} n_{k}-n_{i} m_{k}\right) \mathbf{i}+\left(n_{i} l_{k}-l_{i} n_{k}\right) \mathbf{j}+\left(l_{i} m_{k}-m_{i} l_{k}\right) \mathbf{k}
$$
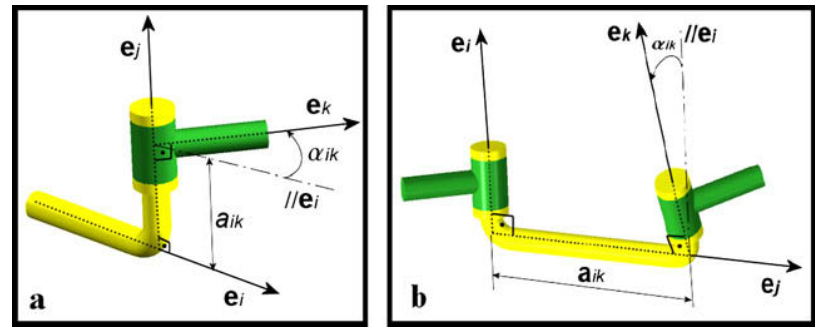

Fig. 1. Joint and link parameters of a spatial link. 
Expanding Eq. (2) yields,

$$
\left.\begin{array}{l}
l_{j} \operatorname{Sin} \alpha_{i k}=0 l_{k}-n_{i} m_{k}+m_{i} n_{k} \\
m_{j} \operatorname{Sin} \alpha_{i k}=n_{i} l_{k}+0 m_{k}-l_{i} n_{k} \\
n_{j} \operatorname{Sin} \alpha_{i k}=-m_{i} l_{k}+l_{i} m_{k}+0 n_{k}
\end{array}\right\} \text { or } \mathbf{e}_{j} \operatorname{Sin} \alpha_{i k}=\mathbf{A} \mathbf{e}_{k}, \quad \text { where } \mathbf{A}=\left[\begin{array}{ccc}
0 & -n_{i} & m_{i} \\
n_{i} & 0 & -l_{i} \\
-m_{i} & l_{i} & 0
\end{array}\right]
$$

If both sides of the Eq. (3) are multiplied by $\mathbf{e}_{j}^{T}$, the result will be

$$
\mathbf{e}_{j}^{T} \mathbf{e}_{j} \operatorname{Sin} \alpha_{i k}=\mathbf{e}_{j}^{T} \mathbf{A} \mathbf{e}_{k} \text { or } \operatorname{Sin} \alpha_{i k}=\mathbf{e}_{i j} \cdot \mathbf{e}_{k}
$$

where,

$$
\mathbf{e}_{j}^{T} \mathbf{e}_{j}=1, \quad \mathbf{e}_{i j}=\mathbf{e}_{j}^{T} \mathbf{A}, \quad \mathbf{e}_{i j}=\left[l_{i j} m_{i j} n_{i j}\right]^{T}, \quad l_{i j}=m_{j} n_{i}-n_{j} m_{i}, \quad m_{i j}=n_{j} l_{i}-l_{j} n_{i}, \quad n_{i j}=l_{j} m_{i}-m_{j} l_{i}
$$

Using Eq. (4), the system of Eq. (1) can be written in the following form,

$$
\mathbf{e}_{i j} \cdot \mathbf{e}_{k}=\operatorname{Sin} \alpha_{i k}, \quad \mathbf{e}_{i} \cdot \mathbf{e}_{k}=\operatorname{Cos} \alpha_{i k}, \quad \mathbf{e}_{j} \cdot \mathbf{e}_{k}=0
$$

Solution of the system of Eq. (5) results in,

$$
\mathbf{g}^{T} \mathbf{B}=\mathbf{e}_{k}
$$

where, $\mathbf{g}=\left[\operatorname{Sin} \alpha_{i k} \operatorname{Cos} \alpha_{i k} 0\right]^{T}, \mathbf{B}=\left[\mathbf{e}_{i j} \mathbf{e}_{\mathbf{i}} \mathbf{e}_{\mathbf{j}}\right]$

Finally Eq. (6) gives us the recurrent unit vector equations as;

$$
l_{k}=l_{i j} \operatorname{Sin} \alpha_{i k}+l_{i} \operatorname{Cos} \alpha_{i k}, \quad m_{k}=m_{i j} \operatorname{Sin} \alpha_{i k}+m_{i} \operatorname{Cos} \alpha_{i k}, \quad n_{k}=n_{i j} \operatorname{Sin} \alpha_{i k}+n_{i} \operatorname{Cos} \alpha_{i k}
$$

Recurrent unit vector equations (Eq. (7)) can be used to describe an orientation of a rigid body with respect to reference frame.

\section{New overconstrained RRRS linkage with linear and angular constraints}

During the history of mechanisms many overconstrained linkages have been discovered and synthesized by using angular conditions. However, in the same period, the number of mechanisms designed by using both angular and linear conditions is relatively scarce and especially they are designed by the combinations of Bennett conditions with other angular constraints.

In the path of a new design of the current study, linear and angular constraints have been created by the analytical approach for the new RRRS linkage shown in Fig. 2. The hinges 1,2 and 3 with arbitrary directions $\mathbf{e}_{2}, \mathbf{e}_{4}$ and $\mathbf{e}_{6}$ are described by joint parameters $\left\{a_{13}, \alpha_{13}\right\},\left\{a_{35}, \alpha_{35}\right\}$, and $\left\{a_{57}, \alpha_{57}\right\}$, so that the remaining link parameters will be $\left\{a_{24}, \alpha_{24}\right\},\left\{a_{46}, \alpha_{46}\right\}$, and $a_{68}$. Note that, the spherical joint $\mathbf{S}$ and the first revolute joint are connected to the fixed frame, parameters $\alpha_{13}, \alpha_{35}$ and $\alpha_{57}$ are variable and remaining parameters are constant. As shown in Fig. 2 the vector loop-closure equation for the mentioned overconstrained mechanism can be written as follows:

$$
\sum_{i=2}^{7} \mathbf{e}_{i} a_{i-1, i+1}=\boldsymbol{\rho}_{c}, \quad \text { where } \boldsymbol{\rho}_{c}=\left[x_{c}, y_{c}, z_{c}\right]^{T}, \quad \mathbf{e}_{i}=\left[l_{i}, m_{i}, n_{i}\right]^{T}
$$

The vector Eq. (8) can also be written in the vector matrix form as,

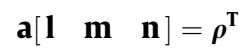

where $\mathbf{a}=\left[a_{i-1, i+1}\right]^{T}, \mathbf{l}=\left[l_{i}\right], \mathbf{m}=\left[m_{i}\right], \mathbf{n}=\left[n_{i}\right], \boldsymbol{\rho}=\left[x_{c} y_{c} z_{c}\right]^{T} i=2, \ldots, 7$.

The vectors $\left\{\mathbf{e}_{i}\right\}_{3}^{7}$ can be calculated by using recurrent unit vector equations (Eq. (7)) with given vectors $\mathbf{e}_{1}=[1,0,0]^{T}$ and $\mathbf{e}_{2}=[0,0,1]^{T}$ as below,

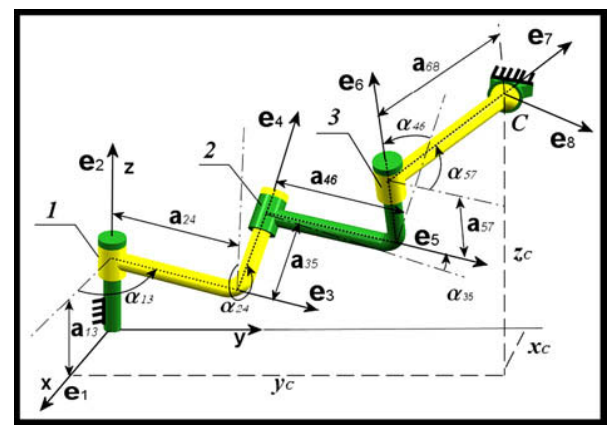

Fig. 2. Parameters for RRRS linkage. 


$$
\begin{aligned}
& \mathbf{e}_{3}=\left(\begin{array}{c}
C_{13} \\
S_{13} \\
0
\end{array}\right), \quad \mathbf{e}_{4}=\left(\begin{array}{c}
S_{13} S_{24} \\
-C_{13} S_{24} \\
C_{24}
\end{array}\right), \quad \mathbf{e}_{5}=\left(\begin{array}{c}
C_{13} C_{35}-C_{24} S_{13} S_{35} \\
S_{13} C_{35}+C_{24} C_{13} S_{35} \\
S_{24} S_{35}
\end{array}\right), \quad \mathbf{e}_{6}=\left(\begin{array}{c}
C_{46} S_{13} S_{24}+\left(C_{24} S_{13} C_{35}+C_{13} S_{35}\right) S_{46} \\
S_{35} S_{13} S_{46}-\left(C_{24} S_{46} C_{35}+C_{46} S_{24}\right) C_{13} \\
C_{24} C_{46}-C_{35} S_{24} S_{46}
\end{array}\right), \\
& \mathbf{e}_{7}=\left(\begin{array}{c}
C_{13}\left(C_{35} C_{57}-C_{46} S_{35} S_{57}\right)-S_{13}\left(-S_{24} S_{46} S_{57}+C_{24}\left(C_{57} S_{35}+C_{35} C_{46} S_{57}\right)\right) \\
C_{57}\left(C_{35} S_{13}+C_{13} C_{24} S_{35}\right)+S_{57}\left(-C_{46} S_{13} S_{35}+C_{13}\left(C_{35} C_{46} C_{24}-S_{24} S_{46}\right)\right) \\
C_{57} S_{24} S_{35}+S_{57}\left(C_{35} S_{24} C_{46}+C_{24} S_{46}\right)
\end{array}\right)
\end{aligned}
$$

where, $C_{i k}$ and $S_{i k}$ represent the cosine and sine of the angle $\alpha_{i k}$ respectively.

Substitution of the elements of the vector values from Eq. (10) into Eq. (9) yields,

$$
\begin{aligned}
& p C_{13}+q S_{13}=x_{c} \\
& p S_{13}-q C_{13}=y_{c} \\
& r+a_{13}=z_{c}
\end{aligned}
$$

where,

$$
\begin{aligned}
& p=a_{24}+C_{35}\left(a_{46}+a_{68} C_{57}\right)+S_{35}\left(a_{57} S_{46}-a_{68} C_{46} S_{57}\right), \\
& q=S_{24}\left(a_{35}+a_{57} C_{46}+a_{68} S_{46} S_{57}\right)-C_{24}\left(S_{35}\left(a_{46}+a_{68} C_{57}\right)+C_{35}\left(a_{68} C_{46} S_{57}-a_{57} S_{46}\right)\right), \\
& r=C_{24}\left(a_{35}+a_{57} C_{46}+a_{68} S_{46} S_{57}\right)+S_{24}\left(S_{35}\left(a_{46}+a_{68} C_{57}\right)+C_{35}\left(a_{68} C_{46} S_{57}-a_{57} S_{46}\right)\right)
\end{aligned}
$$

After this point the following operations are carried on the Eqs. [11-13] for the ease of use. First Eqs. (11) and (12) are multiplied by $S_{13}$ and $C_{13}$ respectively and then subtracted from each other. Second the same equations are multiplied by $C_{13}$ and $S_{13}$ respectively and then added to each other. Finally, after rearranging the Eqs. (11)-(13) the results yield,

$$
\begin{aligned}
& x_{c} S_{13}-y_{c} C_{13}=q \\
& x_{c} C_{13}+y_{c} S_{13}=p \\
& z_{c}-a_{13}=r
\end{aligned}
$$

Expanding Eqs. (15) and (16) with respect to $C_{35}$ and $S_{35}$, we can get the following equations

$$
\begin{aligned}
& p_{1} C_{35}+q_{1} S_{35}=r_{1} \\
& -q_{1} C_{35}+p_{1} S_{35}=r_{2}
\end{aligned}
$$

where $p_{1}=a_{46}+a_{68} C_{57}, q_{1}=a_{57} S_{46}-a_{68} C_{16} S_{57}, r_{1}=x_{c} C_{13}+y_{c} S_{13}-a_{24}$,

$$
r_{2}=z_{c}-a_{13}-C_{24}\left(a_{35}+a_{57} C_{46}+a_{68} S_{46} S_{57}\right)
$$

After solving Eqs. (17) and (18), we will find,

$$
C_{35}=\Delta_{1} \Delta^{-1}=\left|\begin{array}{ll}
r_{1} & q_{1} \\
r_{2} & p_{1}
\end{array}\right|\left|\begin{array}{cc}
p_{1} & q_{1} \\
-q_{1} & p_{1}
\end{array}\right|^{-1}, \quad S_{35}=\Delta_{2} \Delta^{-1}=\left|\begin{array}{cc}
p_{1} & r_{1} \\
-q_{1} & r_{2}
\end{array}\right|\left|\begin{array}{cc}
p_{1} & q_{1} \\
-q_{1} & p_{1}
\end{array}\right|^{-1}
$$

By using $S_{35}$ and $C_{35}$ values found in Eq. (19), a unique value for $\alpha_{35}=A \tan 2\left(S_{35}, C_{35}\right)$ is obtained.

We may consider $S_{i k}$ and $C_{i k}$ as two independent variables and add following trigonometric identities as supplementary equations of constraint,

$$
S_{35}^{2}+C_{35}^{2}=1, \quad S_{57}^{2}+C_{57}^{2}=1, \quad\left(x_{c} S_{13}-y_{c} C_{13}\right)^{2}+\left(y_{c} S_{13}+x_{c} C_{13}\right)^{2}=x_{c}^{2}+y_{c}^{2}
$$

After summing the squares of Eq. (14)-(16) by taking into account Eq. (20) and substituting Eq. (19) into Eq. (14), we obtain the following equations with respect to the unknowns $C_{57}$ and $S_{57}$ as,

$$
\begin{aligned}
& C_{57}=\left[r_{3}-2 a_{35} S_{24}\left(x_{c} S_{13}-y_{c} C_{13}\right)-2 a_{24}\left(y_{c} S_{13}+x_{c} C_{13}\right)\right] p_{2}^{-1} \\
& S_{57}=\left[r_{4}+S_{24}\left(x_{c} S_{13}-y_{c} C_{13}\right)\right] q_{2}^{-1}
\end{aligned}
$$

where $r_{3}=x_{c}^{2}+y_{c}^{2}+\left(z_{c}-a_{13}\right)^{2}+a_{24}^{2}+a_{35}^{2}-a_{46}^{2}-a_{57}^{2}-a_{68}^{2}-2 a_{35}\left(z_{c}-a_{13}\right) C_{24}, r_{4}=C_{24}\left(z_{c}-a_{13}\right)-a_{57} C_{46}-a_{35}, p_{2}=2 a_{46} a_{68}$, and $q_{2}=a_{68} S_{46}$.

Eqs. (21) and (22) both represent a unique solution for $\alpha_{57}=A \tan 2\left(S_{57}, C_{57}\right)$. Due to the fact that $S_{57}$ and $C_{57}$ are found, by using the trigonometric identities (Eq. (20)) we can reach the following overconstraint equation,

$$
4 a_{46}^{2}\left[r_{4}+\alpha S_{24}\right]^{2}+S_{46}\left[r_{3}-2 a_{35} S_{24} \alpha-2 a_{24} \beta\right]^{2}-4 a_{46}^{2} a_{68}^{2} S_{46}^{2}=0
$$

where $\alpha=x_{c} S_{13}-y_{c} C_{13}, \beta=x_{c} C_{13}+y_{c} S_{13}$

Arranging overconstraint Eq. (23), the following polynomial equation can be constructed as,

$$
A \alpha^{2}+B \alpha+C \beta+D \alpha \beta+E=0
$$


where, $\quad A=4 a_{46}^{2} S_{24}^{2}+4 a_{35}^{2} S_{24}^{2} S_{46}^{2}-4 a_{24}^{2} S_{46}^{2}, B=8 a_{46}^{2} r_{4} S_{24}+4 a_{35} r_{3} S_{24} S_{46}^{2}, C=4 a_{24} r_{3} S_{46}^{2}, D=8 a_{24} a_{35} S_{24} S_{46}^{2}, E=4 a_{46}^{2} r_{4}^{2}+S_{46}^{2} r_{3}^{2}-$ $4 a_{46}^{2} a_{68}^{2} S_{46}^{2}+\left(x_{c}^{2}+y_{c}^{2}\right) 4 a_{24}^{2} S_{46}^{2}$.

In the case of mobile mechanism, the functions $\alpha=f\left(\alpha_{13}\right), \beta=g\left(\alpha_{13}\right)$ are not constant, thus coefficients of the polynomial Eq. (24) must be equal to zero $(A=B=C=D=E=0)$. As a result, we will get the following linear and angular conditions for the overconstraint mechanism RRRS as,

$$
\begin{aligned}
& a_{46}^{2} S_{24}^{2}+a_{35}^{2} S_{24}^{2} S_{46}^{2}-a_{24}^{2} S_{46}^{2}=0, \quad 2 a_{46}^{2} r_{4} S_{24}+a_{35} r_{3} S_{24} S_{46}^{2}=0, \quad a_{24} r_{3} S_{46}^{2}=0, \quad a_{24} a_{35} S_{24} S_{46}^{2}=0, \\
& 4 a_{46}^{2} r_{4}^{2}+S_{46}^{2} r_{3}^{2}-4 a_{46}^{2} a_{68}^{2} S_{46}^{2}+\left(x_{c}^{2}+y_{c}^{2}\right) 4 a_{24}^{2} S_{46}^{2}=0
\end{aligned}
$$

Now for simplification, let the joint offset parameters $a_{35}$ be zero. Thus solving Eq. (25) both gives us the linear and angular constraints and the coordinates of the spherical joint for the overconstrained RRRS mechanism with mobility $M=1$.

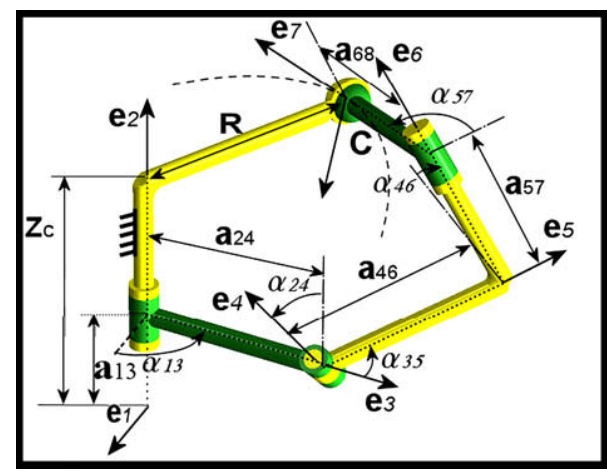

Fig. 3. New 3D overconstraint RRRS linkage with linear and angular constraint.

Table 1

\begin{tabular}{|c|c|c|c|c|c|}
\hline $\begin{array}{l}\text { Structural } \\
\text { bonding }\end{array}$ & $\begin{array}{l}\text { Structural properties } \\
\text { of joint axes }\end{array}$ & Geometry of joint axes & $\begin{array}{l}\text { Structural } \\
\text { bonding }\end{array}$ & Structural properties of joint axes & Geometry of joint axes \\
\hline Angular & & & Linear-angular & & \\
\hline$\overline{\$ \$ \$}$ & Parallel & & $\$ \perp \$ \perp \$$ & Skew perpendicular to each other & \\
\hline$\overline{\$ \$}$ & & & $\$ \perp \$$ & & \\
\hline$(\$ \$ \$)$ & Intersecting in one point & & $\$ \$ \$$ & Arbitrary & \\
\hline$(\$ \$)$ & & & $\$ \$$ & & \\
\hline$\$=\$$ & Coincident & $\therefore$ & & & \\
\hline
\end{tabular}

Structural Bonding of Mechanisms. 


$$
a_{46}=a_{24} S_{46} S_{24}^{-1}, \quad z_{c}=a_{57} C_{46} C_{24}^{-1}+a_{13}, \quad x_{c}^{2}+y_{c}^{2}=R^{2}=a_{46}^{2} a_{68}^{2} a_{24}^{-2}, \quad a_{68}=\left(a_{24}^{2}+a_{57}^{2} \operatorname{Tan}^{2} \alpha_{24}\right)^{0.5}
$$

The new overconstrained RRRS linkage with linear and angular constraints as described by Eq. (26) is introduced in Fig. 3 .

Overconstrained mechanisms are usually created by using either angular conditions, such as intersecting joint axes in one point, joints with parallel axes, and joints with coincident axis or linear and angular conditions, such as joints with skew perpendicular axes and arbitrary axes. The structural bondings of these are illustrated in Table 1.

The common cases of presented analytical approach results are described by four known types of overconstrained RRRS mechanisms with angular and linear-angular conditions as shown in Table 2.

Two new overconstraint RRRS mechanisms are illustrated in Table 2 by using linear and angular conditions with respect to Eq. (26). As represented in Table 2. the fifth one introduces the symmetrical overconstrained RRRS mechanism and the sixth one introduces the asymmetrical RRRS mechanism with linear and angular constraint.

Table 2

Overconstrained RRRS mechanisms with angular and linear-angular conditions. ${ }^{\text {a }}$

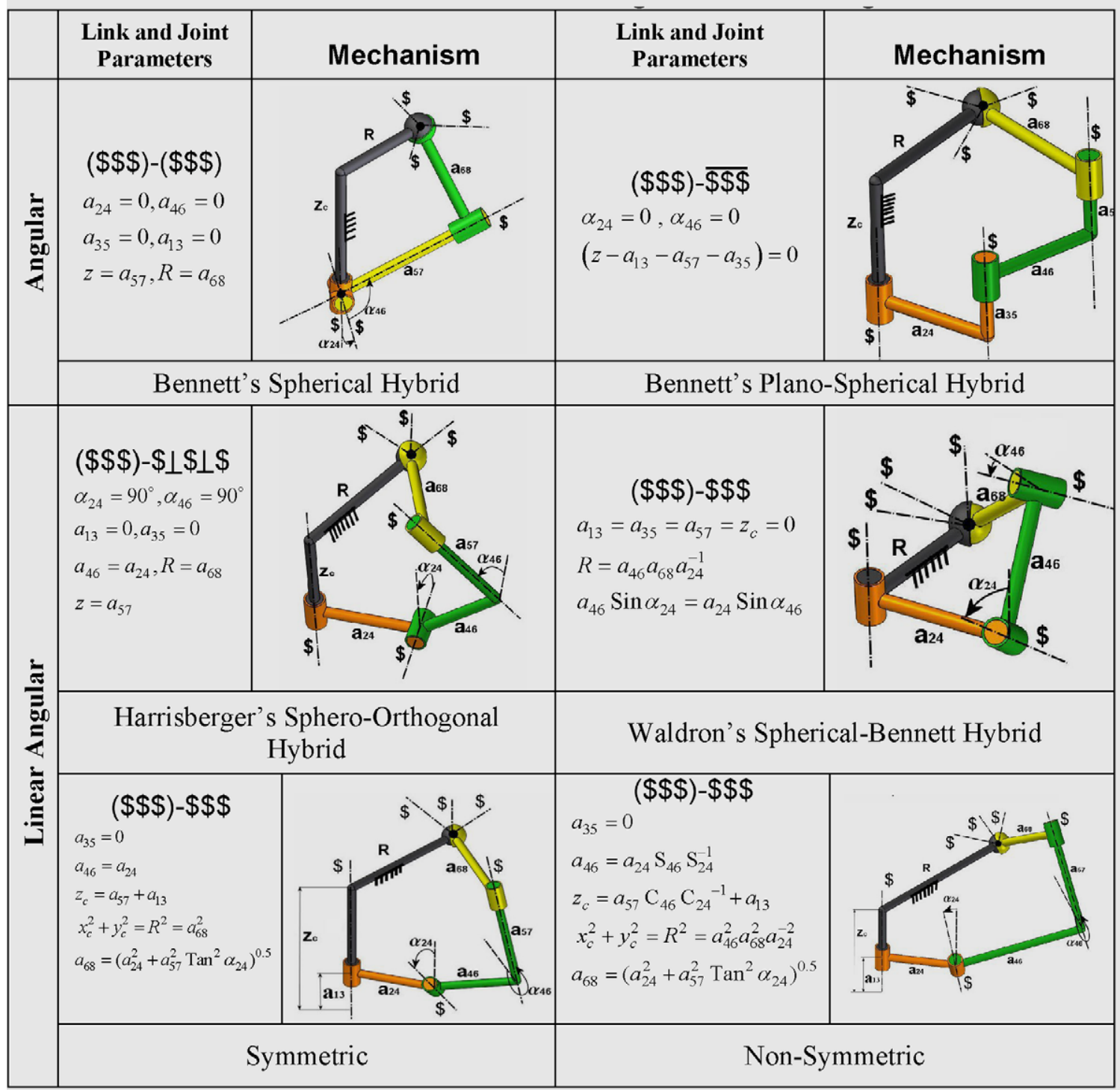

\footnotetext{
${ }^{a}$ The animations of two new overconstrained mechanisms can be seen at www.iyte.edu.tr/ erkingezgin/mechanisms.
} 


\section{Creation of structural groups with general constraint}

In classical definition structural groups are the kinematic chains that have zero mobility. As it is the first main step in structural design of robot manipulators, creation of these groups has vital importance. However, to create such structural groups related with the current subject, the term general constraint should be clearly defined.

In fact, the general constraint of the manipulators refers to the difference between the maximum possible achievable motion of their single link that is assumed to be moving freely in general space $(\lambda=6)$ and the maximum possible achievable free motion of the same link in the space or subspace $(\lambda=5,4,3,2)$, in which the manipulator is actually moving. Note that this definition only valid for the manipulators, where the general constraint is constant.

throughout the manipulator. Due to the fact that, the maximum possible achievable motion of any single link is equal to its space or subspace number $(\lambda)$, the general constraint $(\boldsymbol{d})$ can be formulated as,

$$
\boldsymbol{d}=6-\lambda
$$

where in Eq. (27), 6 represents the general space number, as it is always constant for every manipulator, $\lambda$ is the number of independent scalar equations of closure loop.

Introducing the definition, the general structural mobility formula for the manipulators with general constraint can now be introduced in the following form,

$$
M=(d-6) L+\sum_{i=1}^{j} f_{i}
$$

where $M$ is the mobility of the manipulator, $j$ is the number of joints, $f_{i}$ is the degrees of freedom of the $i$ th joint, and $L$ is the number of independent loops of the manipulator. As the mobility of any structural group is zero, by using Eq. (28), the objective function of the structural groups with general constraint can be given as,

$$
\sum_{i=1}^{j} f_{i}=(6-d) L
$$

If only one DoF pairs to be used in the predesign Eq. (29) will be reduced to

$$
j=(6-d) L
$$

Using the objective function given in Eq. (30), for the joints with one degree of freedom any designer can easily create structural groups for the manipulators with general constraint in the pre-manipulator designs by following the procedure below.

- Determine the moving space or subspace $(\lambda)$ and the number of independent loops $(L)$ of the desired manipulator that will be designed for the specific task.

- Calculate the general constraint (d) of the manipulator by using Eq. (27).

- Calculate the number of joints $(j)$ by using Eq. (30).

- By using appropriate and desired angular and linear-angular conditions of the selected space or subspace, combine the joints together with links to create the structural groups.

Note that, as the current study focuses on parallel manipulators with general constraint one, only the angular and linearangular conditions for $\lambda=5$ will be given in further sections.

\section{Creation of structural groups with general constraint one}

This section describes the creation of the structural groups with general constraint one as well as the descriptions of angular and linear-angular conditions for the subspace $\lambda=5$.

As the parameters, general constraint and the number of loops, are pre-determined $(\boldsymbol{d}=1, L=1)$, the objective function Eq. (30) for the current task will result in the number of joints equals to five $\left(j=\sum f_{i}=5\right.$ ). Calculating the number of joints, the appropriate angular (Table 3 ) and linear-angular conditions (Table 4) should be considered in order to combine the joints with links.

Table 3 shows the four possible angular conditions for $\lambda=5$. The geometry of the condition (Table 3.1) can be created by the rotation of two spheres that have the constant distance between each other. The conditions (Table 3.2) and (Table 3.3) describe the motion of the sphere on the plane or plane on the sphere. And the last condition (Table 3.4) consists of two sets of parallel axes that give us two planes with constant twist angle.

Table 4 demonstrates the eight possible linear-angular conditions for $\lambda=5$. The conditions (Table 4.1) and (Table 4.2) describe the motion of a plane on an elliptic torus and vice versa. The conditions (Table 4.3) through (Table 4.6) can be generated by the motion of a sphere on an elliptic torus and vice versa, where the geometry of the elliptic torus differentiates due to the joint and link parameters. Note that, in conditions (Table 4.5) and (Table 4.6) the elliptic torus shifts in to 
Table 3

Possible angular conditions for the subspace $\lambda=5$.

\begin{tabular}{|c|c|c|c|}
\hline 1 & 2 & & \\
\hline $\begin{array}{l}\text { Two spheres with } \\
\text { constant distance }\end{array}$ & $\begin{array}{c}\text { Sphere on plane } \\
\text { or plane on sphere }\end{array}$ \\
\hline
\end{tabular}

Table 4

Possible linear-angular conditions for the subspace $\lambda=5$.

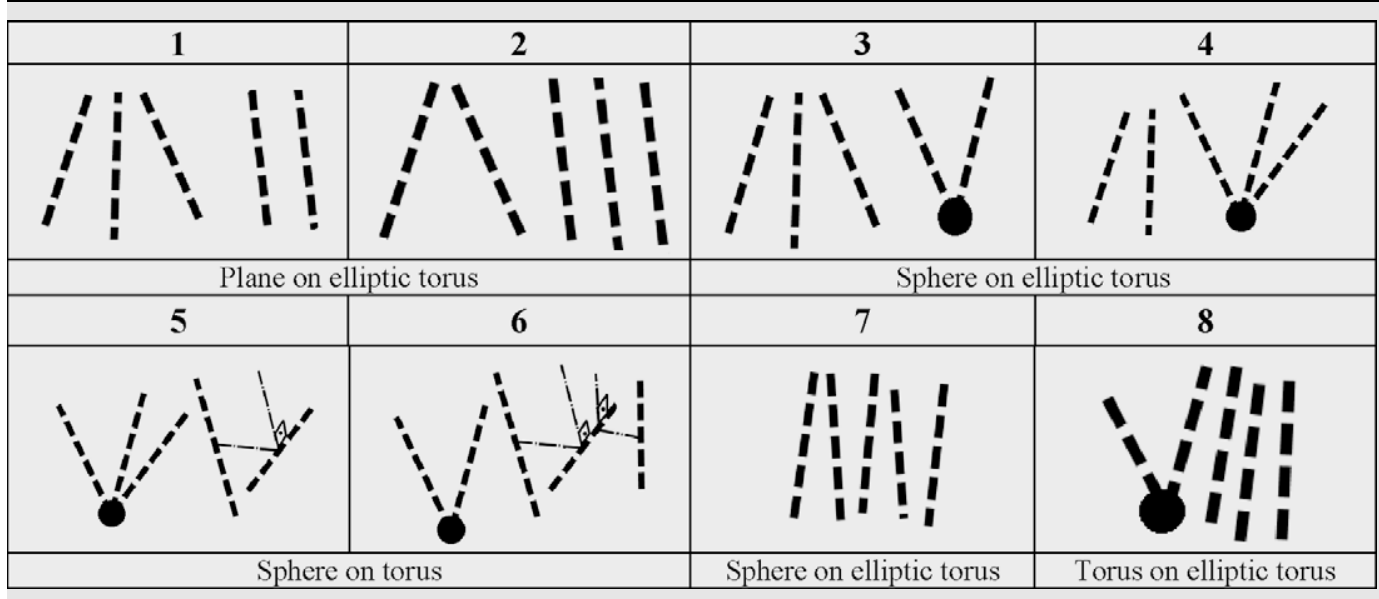

torus because of the perpendicular axes. The condition (Table 4.7) is the basis for the motion of an elliptical torus on an elliptical torus and the last condition (Table 4.8) is similar to the former with one intersection for two of the joint axes.

Finally, using the conditions that are described above, the calculated joints can be combined in 14 possible different ways that results in the structural groups with general constraint one (Table 5).

\section{Mobility of the end effector chains in space or subspaces}

Describing the creation of simple structural groups of robot manipulators with general constraint, an important procedure should be followed to give the desired mobilities to the robot manipulators. In the light of this, creation of the end effector chains gains great importance.

By adding the joints and the branch loops to the end effector that is freely moving in space or any subspace, different end effector chains can be formed (Fig. 4). With respect to their pair and branch loop properties, each of these end effector chains has its own mobility. The formed end effector chains will then be combined appropriately with the zero mobility structural groups to create robot manipulators with various mobilities.

In general, a single end effector moving free in space or any subspace $\lambda$ has mobility equals to the same space or subspace number. However, when connected with pairs and branch loops, mobility of the resultant end effector chain will become,

$$
M_{e}=\lambda_{e}+\sum f_{s}+\sum_{L=1}^{n}\left(f_{L}-\lambda_{L}\right)
$$


Table 5

Simple structural groups with general constraint one respect to angular and linear-angular conditions.

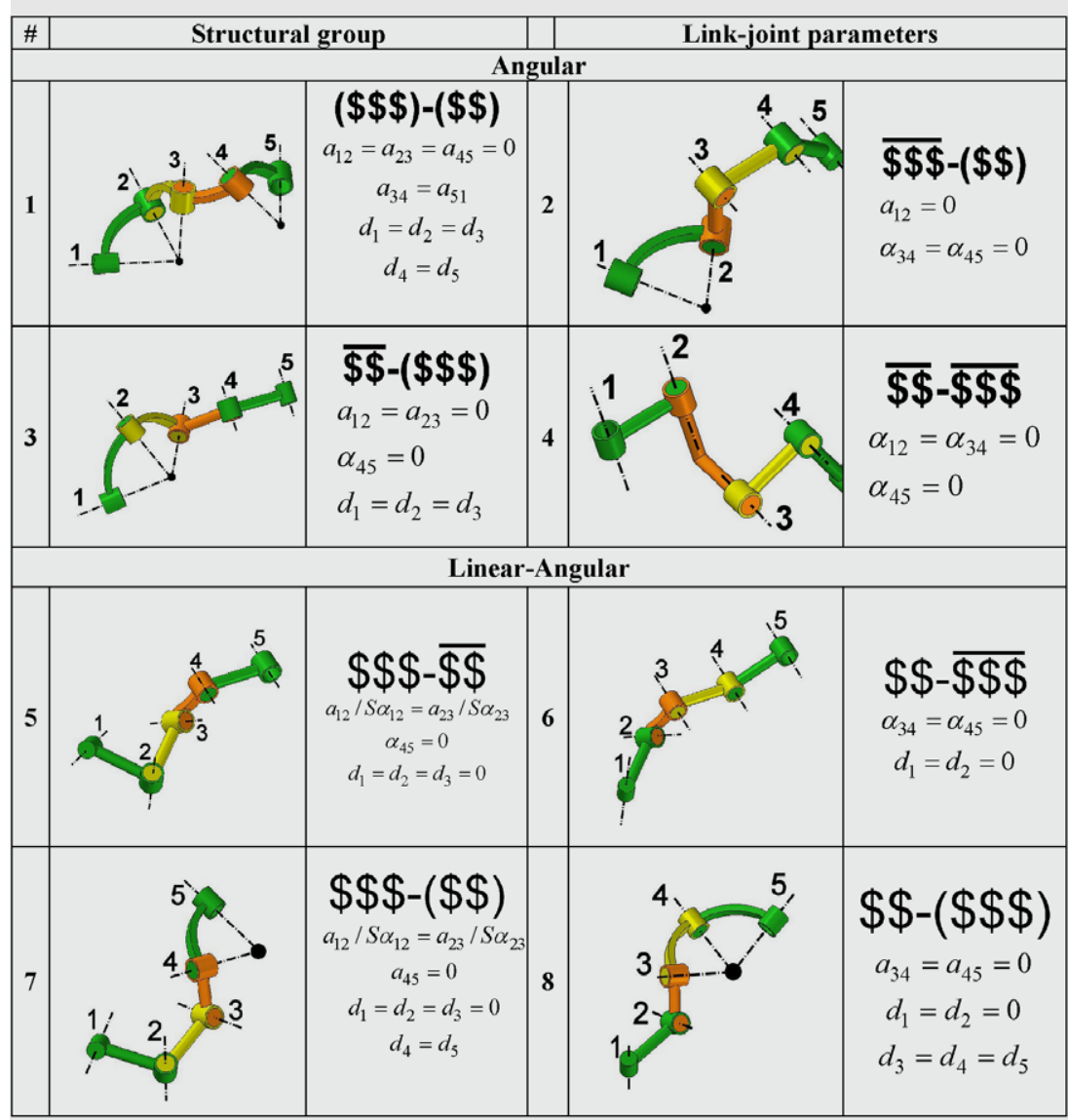

\begin{tabular}{|c|c|c|c|c|c|}
\hline 9 & & $\begin{array}{c}(\$ \$ \$)-\$ \perp \$ \\
a_{12}=a_{23}=0 \\
d_{5}=0 \\
\alpha_{45}=\pi / 2 \\
d_{1}=d_{2}=d_{3}\end{array}$ & 10 & & $\begin{array}{c}(\$ \$)-\$ \perp \$ \perp \$ \\
a_{12}=0 \\
d_{4}=d_{5}=0 \\
\alpha_{34}=\alpha_{45}=\pi / 2 \\
a_{34}=a_{45}\end{array}$ \\
\hline 11 & & $\begin{array}{c}(\$ \$ \$)-\$ \$ \\
a_{12}=a_{23}=0 \\
d_{5}=0\end{array}$ & 12 & & $\begin{array}{c}\$ \$ \$-(\$ \$) \\
d_{1}=d_{2}=0 \\
a_{12} / S \alpha_{12}=a_{23} / S \alpha_{23} \\
a_{34}=\left(a_{23}^{2}+d_{3}^{2} \operatorname{Tan}^{2} \alpha_{12}\right)^{0.5}\end{array}$ \\
\hline 13 & 1 & $\begin{array}{c}\$ \$ \$ \$ \$ \\
d_{1}=d_{2}=d_{3}=0 \\
d_{4}=d_{5}=0 \\
a_{12} / S \alpha_{12}=a_{23} / S \alpha_{23} \\
a_{23}=a_{34}=a_{45}\end{array}$ & 14 & 5 & $\begin{array}{c}(\$ \$)-\$ \$ \$ \\
d_{1}=d_{2}=d_{3}=0 \\
d_{4}=d_{5}=0 \\
a_{12} / S \alpha_{12}=a_{23} / S \alpha_{23} \\
a_{34} / S \alpha_{34}=a_{45} / S \alpha_{45} \\
\alpha_{12}=\pi-\alpha_{34} \\
\alpha_{23}=\pi / 2\end{array}$ \\
\hline
\end{tabular}




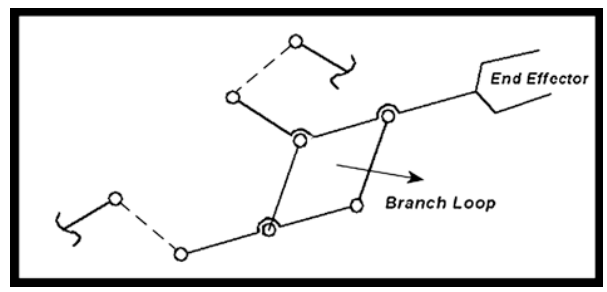

Fig. 4. End effector chain with branch loop.

where, $\lambda_{e}$ is the space or subspace of the end effector, $\sum f_{s}$ is the total degrees of freedom of the single pairs; that is, the pairs outside the branch loops, $n$ is the number of branch loops, $f_{L}$ is the total degrees of freedom of the pairs of the $L$ th branch loop and $\lambda_{L}$ is the space or subspace of the $L$ th branch loop. As it is clear that, $\sum f_{s}=\sum_{i=1}^{k} f_{i}-\sum_{L=1}^{n} f_{L}$ the Eq. (31) will be reduced to,

$$
M_{e}=\lambda_{e}+\sum_{i=1}^{k} f_{i}-\sum_{L=1}^{n} \lambda_{L}
$$

where, $k$ is the number of pairs in the end effector chain.

Finally, after the proper connection between the structural group and the end effector chain, the total mobility of the resultant robot manipulator will become,

$$
M=M_{e}-\lambda+q-j_{p}
$$

where, $\lambda$ is the space or subspace of the base structural group, $j_{p}$ is the number of passive joints and $q$ is the number of redundant links.

\section{Mobility of the end effector chains in subspace $\lambda=5$}

In order to proceed in the design of parallel manipulators with general constraint one, current study considers only the simple end effector chains without branch loops in subspace $\lambda=5$. As there are no branch loops and the subspace of the end effector is $\lambda_{e}=5$, Eq. (32) will be reduced to,

$$
M_{e}=5+\sum_{i=1}^{k} f_{i}
$$

By using Eq. (34), five different end effector chains with various mobilities are created and tabulated to fulfill the design task of the parallel manipulators with general constraint one (Table 6). However, the end effector chains in Table 6 are figured in their simple structural form. Before the connection process with the structural groups is started, they should be reconfigured correctly with respect to the suitable angular or linear-angular conditions of the structural group on which the connection occurs.

\begin{tabular}{|c|c|c|c|c|c|c|c|}
\hline \# & Structure & $\Sigma f_{i}$ & $M_{e}$ & \# & Structure & $\Sigma f_{i}$ & $M_{e}$ \\
\hline 1 & & 0 & 5 & 4 & & 3 & 8 \\
\hline 2 & & 1 & 6 & 5 & & 4 & 9 \\
\hline 3 & & 2 & 7 & 6 & & 5 & 10 \\
\hline
\end{tabular}

Table 6

End effector chains without branch loops in subspace $\lambda=5$. 
Although the figure in Table 6.1 is just an end effector, it is added to the table for the clarification of the step by step development of the end effector chains. Also note that, the maximum mobility of the end effector chains in Table 6 is limited to the $2 \lambda=10$. The reason of the case is the prevention of the redundant motions. Due to the fact that the maximum number of achievable independent motion in subspace $\lambda=5$ is limited to five, 2R3P or 3R2P, after the end effector chains are connected to the structural groups of subspace $\lambda=5$, more than designated mobility $2 \lambda$ will result in the occurrence of the redundant motions with respect to the Eq. (33).

\section{Structural design of the parallel manipulators with general constraint one}

The last and the most important part of this study is the procedure of the structural design in the creation of parallel manipulators with general constraint one by using the results of the previous sections. As the subspace is decided as $\lambda=5$, this procedure can be explained easily by following step by step instructions as,

Table 7

Structural bondings of general constraint one parallel manipulators with angular conditions.

\begin{tabular}{|c|c|c|c|c|c|}
\hline \multirow{2}{*}{$\frac{3}{\frac{3}{2}}$} & \multicolumn{4}{|c|}{ Angular Conditions of Structural groups } & \multirow{2}{*}{ 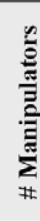 } \\
\hline & a & b & c & $d$ & \\
\hline 2 & 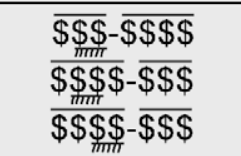 & 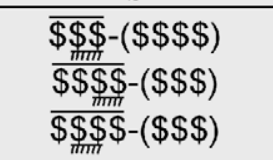 & $\begin{array}{l}(\$ \$ \$)-\overline{\$ \$ \$ \$} \\
(\$ \$ \$ \$)-\overline{\$ \$} \\
(\$ \$ \$ \$)-\overline{\$ \$ \$}\end{array}$ & $\begin{array}{l}(\$ \$ \$)-(\$ \$ \$ \$) \\
(\$ \$ \$ \$)-(\$ \$ \$) \\
(\$ \$ \$ \$)-(\$ \$ \$)\end{array}$ & 12 \\
\hline 3 & 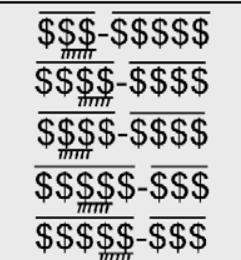 & 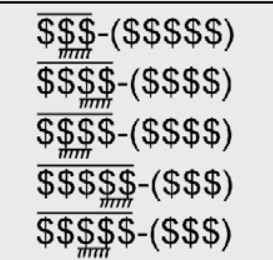 & $\begin{array}{l}(\$ \$ \$)-\overline{\$ \$ \$ \$ \$} \\
(\$ \$ \$ \$)-\overline{\$ \$ \$ \$} \\
(\$ \$ \$ \$)-\$ \$ \$ \$ \\
(\$ \$ \$ \$ \$)-\overline{\$ \$ \$} \\
(\$ \$ \$ \$ \$)-\overline{\$ \$ \$}\end{array}$ & $\begin{array}{l}(\$ \$ \$)-(\$ \$ \$ \$ \$) \\
(\$ \$ \$ \$)-(\$ \$ \$ \$) \\
(\$ \$ \$ \$)-(\$ \$ \$ \$) \\
(\$ \$ \$ \$ \$)-(\$ \$ \$) \\
(\$ \$ \$ \$ \$)-(\$ \$ \$)\end{array}$ & 20 \\
\hline 4 & 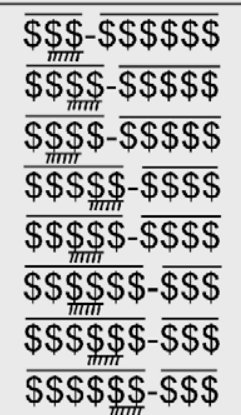 & 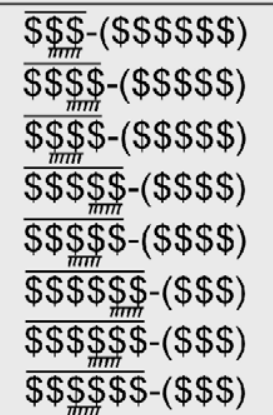 & $\begin{array}{l}(\$ \$ \$)-\overline{\$ \$ \$ \$ \$} \\
(\$ \$ \$ \$)-\overline{\$ \$ \$ \$} \\
(\$ \$ \$ \$)-\$ \$ \$ \$ \$ \\
(\$ \$ \$ \$ \$)-\overline{\$ \$ \$ \$} \\
(\$ \$ \$ \$ \$)-\overline{\$ \$ \$ \$} \\
(\$ \$ \$ \$ \$)-\overline{\$ \$ \$} \\
(\$ \$ \$ \$ \$)-\overline{\$ \$} \\
(\$ \$ \$ \$ \$)-\overline{\$ \$ \$}\end{array}$ & $\begin{array}{l}(\$ \$ \$)-(\$ \$ \$ \$ \$ \$) \\
(\$ \$ \$ \$)-(\$ \$ \$ \$ \$) \\
(\$ \$ \$ \$)-(\$ \$ \$ \$ \$) \\
(\$ \$ \$ \$ \$)-(\$ \$ \$ \$) \\
(\$ \$ \$ \$ \$)-(\$ \$ \$ \$) \\
(\$ \$ \$ \$ \$)-(\$ \$ \$) \\
(\$ \$ \$ \$ \$ \$)-(\$ \$ \$) \\
(\$ \$ \$ \$ \$ \$)-(\$ \$ \$)\end{array}$ & 32 \\
\hline 5 & 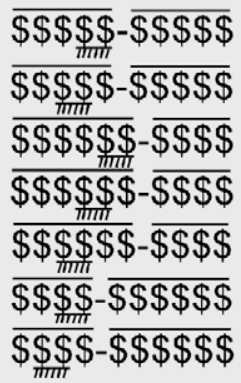 & 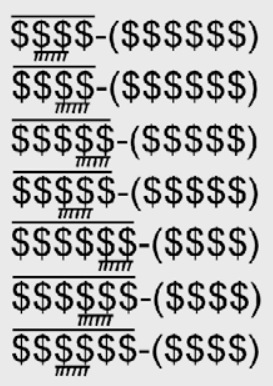 & $\begin{array}{l}(\$ \$ \$ \$)-\overline{\$ \$ \$ \$ \$ \$} \\
(\$ \$ \$ \$)-\overline{\$ \$ \$ \$ \$ \$} \\
(\$ \$ \$ \$ \$)-\overline{\$ \$ \$ \$ \$} \\
(\$ \$ \$ \$ \$)-\overline{\$ \$ \$ \$ \$} \\
(\$ \$ \$ \$ \$ \$)-\overline{\$ \$ \$} \\
(\$ \$ \$ \$ \$ \$)-\overline{\$ \$ \$ \$} \\
(\$ \$ \$ \$ \$ \$)-\overline{\$ \$ \$ \$}\end{array}$ & $\begin{array}{l}(\$ \$ \$ \$)-(\$ \$ \$ \$ \$ \$) \\
(\$ \$ \$ \$)-(\$ \$ \$ \$ \$ \$) \\
(\$ \$ \$ \$ \$)-(\$ \$ \$ \$ \$) \\
(\$ \$ \$ \$ \$)-(\$ \$ \$ \$ \$) \\
(\$ \$ \$ \$ \$ \$)-(\$ \$ \$ \$) \\
(\$ \$ \$ \$ \$ \$)-(\$ \$ \$ \$) \\
(\$ \$ \$ \$ \$ \$)-(\$ \$ \$ \$)\end{array}$ & 28 \\
\hline & 23 & & & 23 & 92 \\
\hline
\end{tabular}


- Decide the desired mobility $(\boldsymbol{M})$ of the manipulator that will be constructed

- Chose one of the conditions of subspace $\lambda=5$ that the manipulator will possess from the Table 3 or Table 4 .

- Select the structural groups from Table 5 with respect to the condition chosen

- Calculate the mobility of the end effector chain $\left(\boldsymbol{M}_{e}\right)$ by using Eq. (33).

- Select the end effector chain from Table 6 that corresponds the mobility $\left(\boldsymbol{M}_{e}\right)$ calculated.

- Combine the selected structural group and the end effector chain in accordance with the chosen condition.

- Select the ground link of the designed parallel manipulator with general constraint one.

Note that Tables 7 and 8 show 163 structural bondings of all possible one loop parallel manipulators with general constraint one that have 92 manipulators with angular and 71 manipulators with linear-angular conditions.

Example: Let us design four overconstrained parallel manipulators with angular and linear-angular conditions.

(A) The first overconstrained manipulators mobility is decided as $\boldsymbol{M}=\mathbf{2}$ with the condition shown in Table 3.4. The structural group with respect to the condition is selected from Table 5.4. The mobility of the end effector chain is calculated as $M_{e}=M+\lambda=2+5=7$ and the end effector chain is selected as Table 6.3. The overconstrained manipulator will

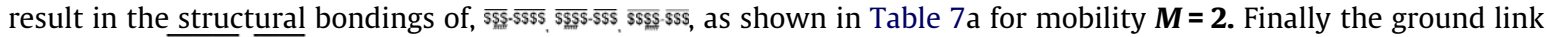
is selected ( $(\overline{\$ \$ \$ \$} \$ \overline{\$ \$}$ ) for construction and the resultant manipulator is shown in Table 9.1.

(B) The second overconstrained manipulators mobility is decided as $\boldsymbol{M}=\mathbf{4}$ with the condition shown in Table 3.3. The structural group with respect to the condition is selected from Table 5.3. The mobility of the end effector chain is calculated as $M_{e}=M+\lambda=4+5=9$ and the end effector chain is selected as Table 6.5. The overconstrained manipulator will result in the structural bondings as shown in Table $7 \mathrm{~b}$ for mobility $\boldsymbol{M}=\mathbf{4}$. Finally the ground link is selected (\$\$\$\$ $\$$ - $(\$ \$ \$)$ ) for construction and the resultant manipulator is shown in Table 9.2.

Table 8

Structural bondings of general constraint one parallel manipulators with linear-angular conditions

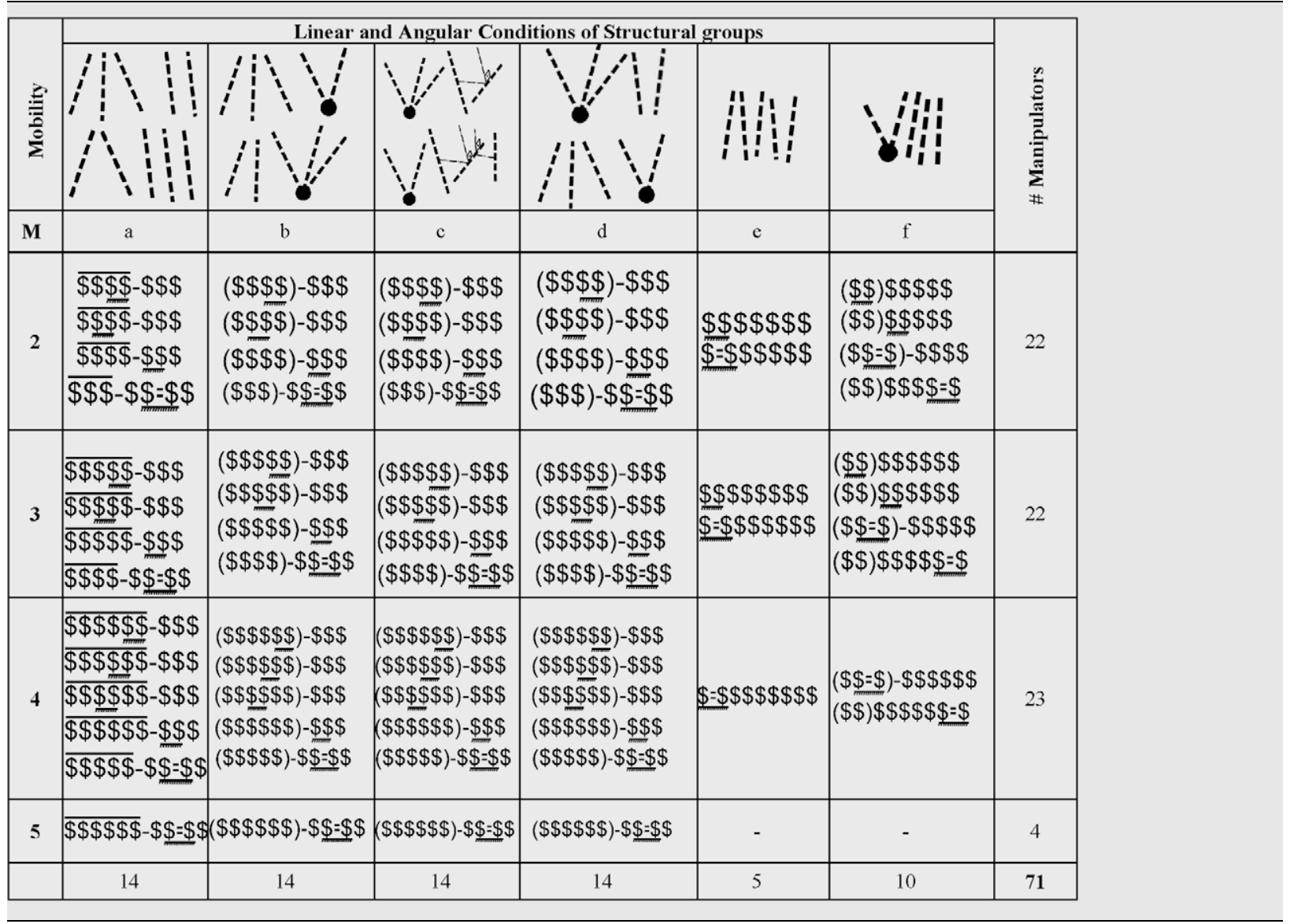


Table 9

New parallel manipulators with general constraint one.

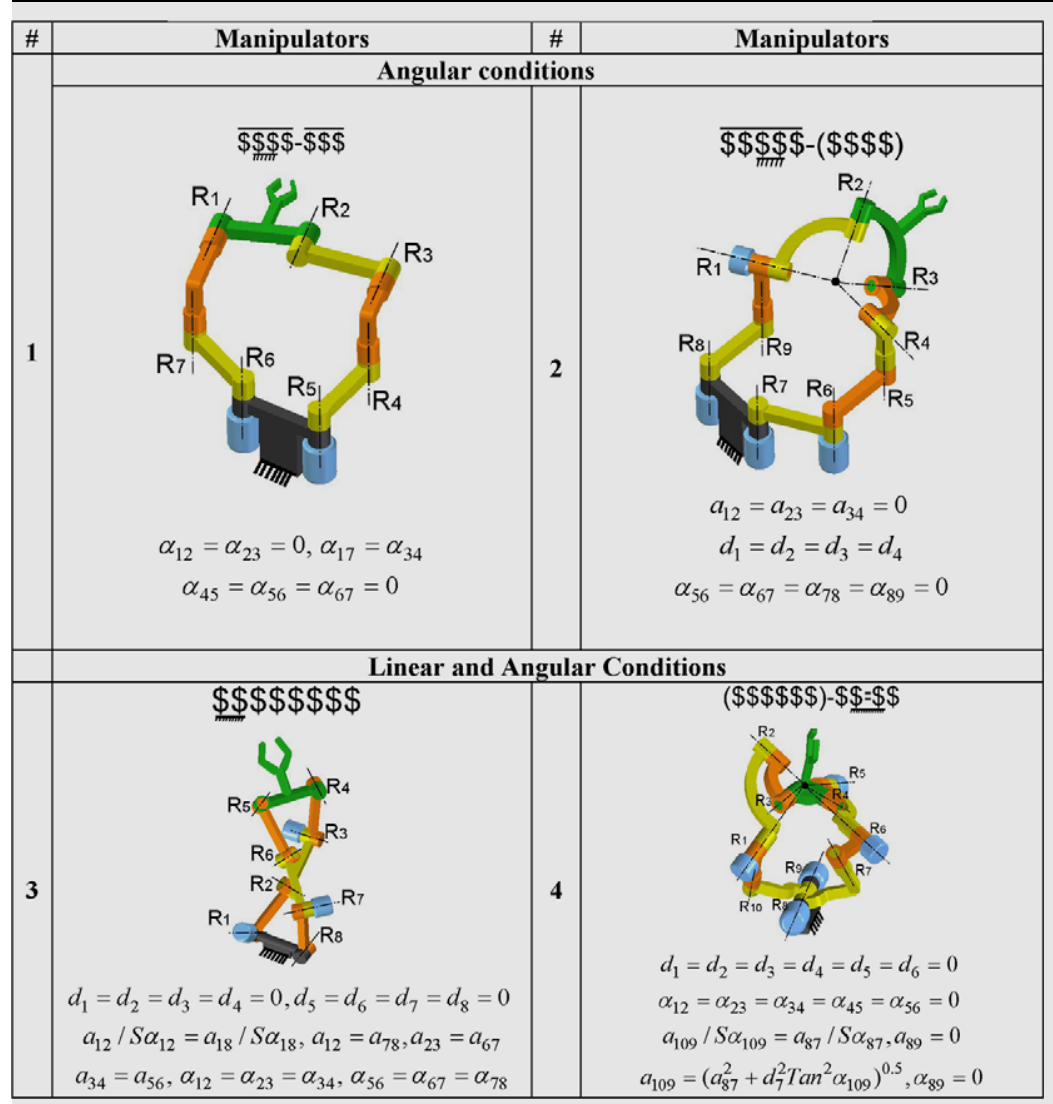

(C) The third overconstrained manipulators mobility is decided as $\boldsymbol{M}=\mathbf{3}$ with the condition shown in Table 4.7. The structural group with respect to the condition is selected from Table 5.13. The mobility of the end effector chain is calculated as $M_{e}=M+\lambda=3+5=8$ and the end effector chain is selected as Table 6.4. The overconstrained manipulator will result in the structural bondings as shown in Table 8 e for mobility $\boldsymbol{M}=\mathbf{3}$. Finally the ground link is selected

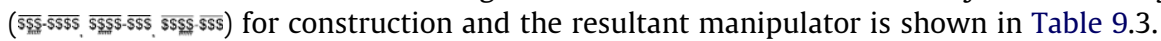

(D) The fourth overconstrained manipulators mobility is decided as $\boldsymbol{M}=\mathbf{5}$ with the condition shown in Table 4.3. The structural group with respect to the condition is selected from Table 5.12. The mobility of the end effector chain is calculated as $M_{e}=M+\lambda=5+5=10$ and the end effector chain is selected as Table 6.6. The overconstrained manipulator will result in the structural bondings as shown in Table $8 \mathrm{~d}$ for mobility $\boldsymbol{M}=\mathbf{5}$. Finally the ground link is selected $((\$ \$ \$ \$ \$)-\$ \$ \$ \$)$ for construction and the resultant manipulator is shown in Table 9.4.

\section{Conclusions}

Throughout the study, structural design of parallel manipulators with general constraint one with respect to the angular and the linear-angular conditions are described as well as the general procedure of describing the orientation of rigid body with respect to the reference frame by writing recurrent unit vector equations. Also new formulations and definitions of constructing overconstrained manipulators are introduced dealing with 14 structural groups with general constraint one and end effector chains in subspace $\lambda=5$ with various mobilities. As a result of the current study, new general constraint one RRRS mechanisms that have linear-angular conditions are introduced. Moreover the proposed method was checked on four known overconstrained mechanisms with angular conditions. By using 14 overconstrained structural groups, all architectures of parallel manipulators with general constraint one that are composed of only revolute joints and single-loop are introduced by using structural bonding and four of them are illustrated along with examples. 


\section{Appendix A. Supplementary data}

Supplementary data associated with this article can be found, in the online version, at doi:10.1016/ j.mechmachtheory.2009.06.004.

\section{References}

[1] P.T. Sarrus, On the transformation of alternative movements rectilinear, circular motions, and conversely, Comptes Rendus Weekly meetings of the Academy of Sciences (1853) 1036-1038.

[2] G.T. Bennett, The parallel motion of Sarrus and some allied mechanisms, Philosophical Magazine, 6th Series 9 (1905) $803-810$.

[3] R. Bricard, Kinematic Lessons, vol. 2, Gauthier-Villars, Paris, 1927, pp. 7-12.

[4] M. Goldberg, New five-bar and six-bar linkages in three dimensions, Transactions of the ASME 65 (1943) 649-663.

[5] R. Franke, From the construction of the gearbox, the German engineering, Düsseldorf (1951) 97-106.

[6] P.G. Altmann, Communications to Grodzinski, P. and M'Ewen, E., link mechanisms in modern kinematics, in: Proceedings of the Institution of Mechanical Engineers 168 (37) (1954) 889-896.

[7] L. Harrisberger, A.H. Soni, A survey of three dimensional mechanisms with general constraint one, ASME, 1966, No. 66-Mech-44.

[8] K.J. Waldron, A family of overconstrained linkages, Journal of Mechanisms 2 (1967) 201-211.

[9] K.J. Waldron, Hybrid overconstrained linkages, Journal of Mechanisms 3 (1968) 73-78.

[10] K.J. Waldron, Symmetric overconstrained linkages, Transactions of ASME, Journal of Engineering for Industry B91 (1969) 158-164.

[11] K. Wohlhart, A new 6R space mechanism, in: Proceedings of the Seventh World Congress on the Theory of Machines and Mechanisms, Sevilla, Spain, vol. 1, 1987, pp. 193-198.

[12] K. Wohlhart, Merging two general Goldberg 5R linkages to obtain a new 6R space mechanism, Mechanism and Machine Theory 26 (2) (1991) 659-668.

[13] C. Mavroidis, B. Roth, New and revised overconstrained mechanisms, Transactions of ASME (117) (1995) 75-82.

[14] P. Dietmeier, A new 6R space mechanism, in: Proceedings of Ninth World Congress on the Theory of Machines and Mechanisms, Milano, vol. 1, 1995, pp. 52-56.

[15] P. Schatz, Rhythm Research and Technology, 2th Circulation, Verlag Freies Geistesleben, Stuttgart, 1998.

[16] R.I. Alizade, F.C. Can, E. Gezgin, O. Selvi, Structural synthesis of new parallel and serial platform manipulators, in: 12th IFToMM World Congress, Besancon, France, June 18-21, 2007.

[17] J.E. Baker, A geometrico-algebraic exploration of Altmann's linkage, Mechanism and Machine Theory 28 (2) (1993) 249-260.

[18] J.E. Baker, The single screw reciprocal to the general plane-symmetric six screw linkage, Journal for Geometry and Graphics (1) (1997) 5-12.

[19] J. Eddie Baker, On equivalent motions in spatial linkages, Mechanism and Machine Theory 35 (2000) 1641-1649.

[20] J. Eddie Baker, Displacement-closure equations of the unspecialised double-Hooke's-joint linkage, Mechanism and Machine Theory 37 (2002) $1127-$ 1144.

[21] J. Eddie Baker, Overconstrained six-bars with parallel adjacent joint-axes, Mechanisms and Machine Theory 38 (2003) $103-117$.

[22] J. Eddie Baker, A curious new family of overconstrained six-bars, Transactions of ASME (127) (2005) 602-606.

[23] C. Mavroidis, B. Roth, Analysis and synthesis of overconstrained mechanisms, in: Proceedings of the ASME Design Technical Conferences, 1994, DE-70, pp. 115-113.

[24] C. Mavroidis, B. Roth, Analysis of overconstrained mechanisms, Transactions of ASME (117) (1995) 69-74.

[25] C. Mavroidis, B. Roth, Method to determine uncertain configurations of 6R manipulators, in: Proceedings of the 9th World Congress on the Theory of Machines and Mechanisms (IFToMM), Milano, Italy, vol. 3., 1995, pp. 1987-1992.

[26] C. Mavroidis, M. Beddows, A spatial overconstrained mechanism that can be used in practical applications, in: Proceedings of the Fifth Applied Mechanisms and Robotics Conference, Cincinnati, OH, October 1997.

[27] A. Karger, Classification of 5R closed kinematic chains with self mobility, Mechanism and Machine Theory 33 (1998) $213-222$

[28] A.J. Shih, H.S. Yan, Synthesis of a single-loop overconstrained six revolute joint spatial mechanism for two-position cylindrical rigid body quidance, Mechanism and Machine Theory 37 (2002) 61-73.

[29] J. Lerbet, Coordinate-free kinematic analysis of overconstrained mechanisms with mobility one Z. angew, Math. Mech 85 (10) (2005) 740-747.

[30] Q. Jin, T. Yang, Overconstraint analysis on spatial 6-link loops, Mechanism and Machine Theory 37 (2002) $267-278$.

[31] J.S. Dai, Z. Huang, H. Lipkin, Mobility of overconstrained parallel mechanisms, Transactions of ASME (128) (2006) $220-229$.

[32] S.D. Guest, P.W. Fowler, A symmetry-extended mobility rule, Mechanism and Machine Theory 40 (2005) $1002-1014$.

[33] C. Huang, C.C. Sun, An investigation of screw systems in the finite displacements of Bennett-based 6R linkages, Transactions of ASME (122) (2000) 426430.

[34] W. Li, J. Sun, J. Zhang, K He, R. Du, A parallel 2 dof spherical mechanism with one-to-one input-output mapping, in: Proceedings of the Sixth WSEAS International Conference on Robotics Control and Manufacturing Technology, Hangzo, China, April 16-18, 2006, pp. 6-11.

[35] G. Gogu, Fully isontropic overconstrained parallel wrists with two degrees of freedom, in: Proceedings of the 2005 IEEE International Conference on Robotics and Automation, Barcelona, Spain, April 2005, pp. 4014-4019.

[36] J. Denavit, R.S. Hartenberg, A kinematic notation for lower pair mechanisms based on matrices, Journal of Applied Mechanics 22 (1955) 215-221. 